\title{
CROSSED PRODUCTS BY FINITE GROUP ACTIONS WITH THE ROKHLIN PROPERTY
}

\author{
HIROYUKI OSAKA AND N. CHRISTOPHER PHILLIPS
}

\begin{abstract}
We prove that a number of classes of separable unital $\mathrm{C}^{*}$-algebras are closed under crossed products by finite group actions with the Rokhlin property, including:

- AI algebras, AT algebras, and related classes characterized by direct limit decompositions using semiprojective building blocks.

- Simple unital AH algebras with slow dimension growth and real rank zero.

- $\mathrm{C}^{*}$-algebras with real rank zero or stable rank one.

- Simple $\mathrm{C}^{*}$-algebras for which the order on projections is determined by traces.

- $\mathrm{C}^{*}$-algebras whose quotients all satisfy the Universal Coefficient Theorem.

- $\mathrm{C}^{*}$-algebras with a unique tracial state.

Along the way, we give a systematic treatment of the derivation of direct limit decompositions from local approximation conditions by homomorphic images which are not necessarily injective.
\end{abstract}

\section{INTRODUCTION}

The purpose of this paper is to prove theorems of the following type. Let $A$ be a separable unital $\mathrm{C}^{*}$-algebra, let $G$ be a finite group, and let $\alpha: G \rightarrow \operatorname{Aut}(A)$ be an action which has the Rokhlin property. (See Definition 3.1 below.) Suppose that $A$ belongs to a class of $\mathrm{C}^{*}$-algebras characterized by some structural property, such as the AI algebras. Then $C^{*}(G, A, \alpha)$ belongs to the same class. The classes we consider include:

- $\mathrm{C}^{*}$-algebras with various kinds of direct limit decompositions involving semiprojective building blocks.

- Simple unital AH algebras with slow dimension growth and real rank zero.

- $\mathrm{C}^{*}$-algebras with real rank zero or stable rank one.

- Simple $\mathrm{C}^{*}$-algebras for which the order on projections is determined by traces.

- $\mathrm{C}^{*}$-algebras $A$ such that all quotients $A / I$ satisfy the Universal Coefficient Theorem.

- $\mathrm{C}^{*}$-algebras with a unique tracial state.

(For a complete list, see the statements of the theorems in Sections 3 and 4) In most of these classes, the $\mathrm{C}^{*}$-algebras need not be simple.

Date: 6 Feb. 2009.

2000 Mathematics Subject Classification. Primary 46L55; Secondary 46L35.

Research of the first author partially supported by The Open Research Center Project for Private Universities: matching fund from MEXT, 2004-2008. Research of the second author partially supported by NSF grants DMS 0302401 and DMS 0701076. 
The essential observation (except for the last item) is already in the proof of Theorem 2.2 of [28]: the crossed product $C^{*}(G, A, \alpha)$ has a local approximation property by $\mathrm{C}^{*}$-algebras stably isomorphic to homomorphic images of $A$. For some of the classes we consider, this is already enough. For others, such as classes of direct limits involving semiprojective building blocks, technical difficulties arise because we must apparently allow arbitrary homomorphic images in the approximation property. (Homomorphic images of semiprojective $\mathrm{C}^{*}$-algebras need not be semiprojective.) We therefore present a systematic framework for proving that a $\mathrm{C}^{*}$-algebra with a local approximation property by homomorphic images of a suitable class of semiprojective $\mathrm{C}^{*}$-algebras can in fact be written as a direct limit of algebras in the class. Our result is an analog of Lemma 15.2.2 of [21], where the local approximation property uses injective homomorphic images. Results of this kind for specific cases are already implicit in the literature; our contribution is primarily to systematize the method.

We know of only three results like those above in the literature: for AF algebras (Theorem 2.2 of [28]), for approximately divisible $\mathrm{C}^{*}$-algebras, and for $D$-absorbing $\mathrm{C}^{*}$-algebras for appropriate $D$ (both in Corollary 3.4 of [15]). (Some others, for example involving the ideal and projection properties, will appear in 26 . Some of them were proved before this paper was written, but do not use the ideas of this paper.) It is surprising to us that Theorem 2.2 of [28] had not been proved long ago, and even in [28] the much wider applicability of the argument was overlooked.

Our main applications will appear in 25], where we use results of the type presented here to show that various actions of finite groups do not have the Rokhlin property. (Indeed, the Rokhlin property is rather rare.) We present the results of this paper separately for three reasons. First, the main ideas are somewhat different from those in [25]. Second, some of the consequences, such as those involving the Universal Coefficient Theorem and direct limits using infinite building blocks, have no connection with 25. Third, some of the results have other applications. See [30].

In Section 1. we introduce flexible classes of $\mathrm{C}^{*}$-algebras, and prove that if a separable unital $\mathrm{C}^{*}$-algebra has local approximation by homomorphic images of $\mathrm{C}^{*}$-algebras in a flexible class, then it is a direct limit of algebras in the class. The algebras involved need not be simple. In Section 2, we prove that many of the standard classes of semiprojective building blocks are flexible. The most complicated case is the one dimensional noncommutative CW complexes of [12]. Sections 3 and 4 contain the proofs of the closure results under crossed products. They are roughly divided into membership in various classes considered in the Elliott program (Section 3) and more general properties such as stable rank (Section 4).

We take $\mathbb{N}$ to be $\{1,2, \ldots\}$.

\section{DiRECT Limits AND LOCAL APPROXIMATION}

The purpose of this section is to give a uniform description of the local approximation characterizations of the classes of direct limit algebras constructed using some common families of semiprojective building blocks. We do not require our direct limits to either be simple or have real rank zero, and we do not require the maps in our direct systems or local approximations to be injective. The results are known for various specific classes of building blocks.

Definition 1.1. Let $\mathcal{C}$ be a class of separable unital $\mathrm{C}^{*}$-algebras. Then $\mathcal{C}$ is finitely saturated if the following closure conditions hold: 
(1) If $A \in \mathcal{C}$ and $B \cong A$, then $B \in \mathcal{C}$.

(2) If $A_{1}, A_{2}, \ldots, A_{n} \in \mathcal{C}$ then $\bigoplus_{k=1}^{n} A_{k} \in \mathcal{C}$.

(3) If $A \in \mathcal{C}$ and $n \in \mathbb{N}$, then $M_{n}(A) \in \mathcal{C}$.

(4) If $A \in \mathcal{C}$ and $p \in A$ is a nonzero projection, then $p A p \in \mathcal{C}$.

Moreover, the finite saturation of a class $\mathcal{C}$ is the smallest finitely saturated class which contains $\mathcal{C}$.

If one wants to deal with sets instead of classes, one should omit Condition (11).

Definition 1.2. Let $\mathcal{C}$ be a class of separable unital $\mathrm{C}^{*}$-algebras. We say that $\mathcal{C}$ has approximate quotients if:

(1) For every $A \in \mathcal{C}$ and every ideal $I \subset A$, there is an increasing sequence $I_{0} \subset I_{1} \subset \cdots$ of ideals in $A$ such that $\overline{\bigcup_{n=0}^{\infty} I_{n}}=I$, and such that for every $n$ the $\mathrm{C}^{*}$-algebra $A / I_{n}$ is in the finite saturation of $\mathcal{C}$.

We say that $\mathcal{C}$ is flexible if in addition:

(2) For every $A \in \mathcal{C}$, every $n \in \mathbb{N}$, and every nonzero projection $p \in M_{n}(A)$, the corner $p M_{n}(A) p$ is finitely generated, and is semiprojective in the sense of Definition 14.1.3 of [21].

We would like to replace Condition (2) by the simpler requirement that all algebras in $\mathcal{C}$ be semiprojective. However, we do not know whether corners in semiprojective algebras are necessarily semiprojective (although this is true for full corners, by Proposition 2.27 of [2]).

The finite generation condition is technically important in our proofs, but we do not know if it is really necessary. It is unfortunately not true that a corner in a unital finitely generated $\mathrm{C}^{*}$-algebra is again finitely generated. Let $X$ be the Hilbert cube $[0,1]^{\mathbb{N}}$, and let $A=[K \otimes C(X)]^{+}$. By Theorem 8 of [23, the algebra $K \otimes C(X)$ is generated by a single element, so $A$ is finitely generated. However, $A$ has a corner isomorphic to $C(X)$. If $C(X)$ were generated by $n$ elements, we would have an injective map $X \rightarrow \mathbb{C}^{n}$.

The collection of all finite dimensional $\mathrm{C}^{*}$-algebras is finitely saturated and flexible. Further examples will be given in Section 2 .

Lemma 1.3. Let $\mathcal{C}$ be a class of separable unital $\mathrm{C}^{*}$-algebras. If $\mathcal{C}$ is flexible, then its finite saturation is also flexible. If $\mathcal{C}$ satisfies either condition of Definition 1.2 separately, then so does its finite saturation.

Proof. We can obtain the finite saturation of $\mathcal{C}$ by first forming all finite matrix algebras over all algebras in $\mathcal{C}$, then forming all corners of the algebras we have so far, then forming all finite direct sums of the resulting algebras, and finally closing under the isomorphism relation.

It is easy to prove that these operations all preserve Condition (2) of Definition 1.2, using the following three facts: a finite direct sum of semiprojective $\mathrm{C}^{*}$ algebras is semiprojective (Theorem 14.2.1 of [21]), a finite direct sum of finitely generated $\mathrm{C}^{*}$-algebras is finitely generated (easy), and a corner in a finite direct sum is a finite direct sum of corners in the summands.

Condition (11) of Definition 1.2 is obviously preserved under finite direct sums and tensoring with $M_{n}$. We therefore need only show that it passes to corners.

Accordingly, let $A$ be a separable unital $\mathrm{C}^{*}$-algebra satisfying Condition (1), let $p \in A$ be a nonzero projection, and let $I \subset p A p$ be an ideal. Define $J=\overline{A I A}$, 
which is an ideal in $A$. Note that

$$
p J p=\overline{(p A)(p I p)(A p)}=\overline{(p A p) I(p A p)} \subset I .
$$

It follows that $J \cap p A p=p J p=I$.

By hypothesis, there are ideals $J_{0} \subset J_{1} \subset \cdots$ in $A$ such that $\overline{\bigcup_{n=0}^{\infty} J_{n}}=J$ and such that $A / J_{n}$ is in the finite saturation of $\mathcal{C}$ for all $n$. Define $I_{n}=p J_{n} p$, which is an ideal in $p A p$. Note that $I_{n}=J_{n} \cap p A p \subset J \cap p A p=I$. Also,

$$
\overline{\bigcup_{n=0}^{\infty} I_{n}}=\overline{\bigcup_{n=0}^{\infty} p J_{n} p}=p\left(\overline{\bigcup_{n=0}^{\infty} J_{n}}\right) p=p J p=I .
$$

Let $\pi: A \rightarrow A / J_{n}$ and $\kappa: p A p \rightarrow p A p / I_{n}$ be the quotient maps. We next claim that $p A p / I_{n} \cong \pi(p)\left(A / J_{n}\right) \pi(p)$. Since $J_{n} \cap p A p=I_{n}$, we have an obvious injective map $\varphi: p A p / I_{n} \rightarrow A / J_{n}$. For surjectivity, let $x \in \pi(p)\left(A / J_{n}\right) \pi(p)$. Choose $a \in A$ such that $\pi(a)=x$. Then $a-p a p \in J_{n}$. So $\varphi(\kappa($ pap $))=\pi($ pap $)=\pi(a)=x$. This proves the claim.

Since $p A p / I_{n}$ is a corner of $A / J_{n}$, and since $A / J_{n}$ is in the finite saturation of $\mathcal{C}$, it follows that $p A p / I_{n}$ is in the finite saturation of $\mathcal{C}$. This completes the verification that $p A p$ satisfies Condition (11), and the proof of the lemma.

Definition 1.4. Let $\mathcal{C}$ be a class of separable unital $\mathrm{C}^{*}$-algebras. A unital approximate $\mathcal{C}$-algebra is a $\mathrm{C}^{*}$-algebra $A$ which is isomorphic to a direct $\operatorname{limit} \lim A_{n}$ in which each $A_{n}$ is in the finite saturation of $\mathcal{C}$ and in which each homomorphism $\varphi_{n}: A_{n} \rightarrow A_{n+1}$ is unital (but not necessarily injective).

Definition 1.5. Let $\mathcal{C}$ be a class of separable unital $\mathrm{C}^{*}$-algebras. A unital local $\mathcal{C}$-algebra is a separable unital $\mathrm{C}^{*}$-algebra $A$ such that for every finite set $S \subset A$ and every $\varepsilon>0$, there is a $C^{*}$-algebra $B$ in the finite saturation of $\mathcal{C}$ and a unital homomorphism $\varphi: B \rightarrow A$ (not necessarily injective) such that $\operatorname{dist}(a, \varphi(B))<\varepsilon$ for all $a \in S$. If one can always choose $B \in \mathcal{C}$, rather than merely in its finite saturation, we call $A$ a unital strong local $\mathcal{C}$-algebra

Several of the following closure properties will be needed later. Note that, if $\mathcal{C}$ has approximate quotients (Definition 1.2), then the class of unital local $\mathcal{C}$-algebras is finitely saturated.

Lemma 1.6. Let $\mathcal{C}$ be a class of separable unital $\mathrm{C}^{*}$-algebras. Let $\mathcal{L}$ be the class of unital local $\mathcal{C}$-algebras. Then:

(1) If $A_{1}, A_{2}, \ldots, A_{n} \in \mathcal{L}$ then $\bigoplus_{k=1}^{n} A_{k} \in \mathcal{L}$.

(2) If $A \in \mathcal{L}$ and $n \in \mathbb{N}$, then $M_{n}(A) \in \mathcal{L}$.

(3) If $A \in \mathcal{L}$ and $I$ is an ideal in $A$, then $A / I \in \mathcal{L}$.

(4) If $A$ is a unital strong local $\mathcal{L}$-algebra, then $A \in \mathcal{L}$.

Suppose, in addition, that $\mathcal{C}$ has approximate quotients (Definition 1.2). Then:

(5) If $A \in \mathcal{L}$ and $p \in A$ is a nonzero projection, then $p A p \in \mathcal{L}$.

(6) If $A$ is a unital local $\mathcal{L}$-algebra, then $A \in \mathcal{L}$.

Proof. The first four parts are obvious.

For Part (5), without loss of generality $\mathcal{C}$ is finitely saturated. Let $A \in \mathcal{L}$, let $p \in A$ be a nonzero projection, let $S \subset p A p$ be finite, and let $\varepsilon>0$. Set $M=\sup _{a \in S}\|a\|$. Choose $\delta>0$ with

$$
\delta \leq \min \left(1, \frac{\varepsilon}{2 M+3}\right)
$$


and so small that whenever $A$ is a unital $\mathrm{C}^{*}$-algebra, $B \subset A$ is a subalgebra, and $p \in A$ is a projection such that $\operatorname{dist}(p, B)<\delta$, then there are a projection $q \in B$ and a unitary $u \in A$ such that

$$
u q u^{*}=p \quad \text { and } \quad\|u-1\|<\frac{\varepsilon}{2 M+3} .
$$

Write $S=\left\{a_{1}, a_{2}, \ldots, a_{n}\right\}$. Choose $B_{0} \in \mathcal{C}$ and a unital homomorphism $\rho_{0}: B_{0} \rightarrow A$ such that there are $x, c_{1}, c_{2}, \ldots, c_{n} \in B_{0}$ with $\left\|\rho_{0}(x)-p\right\|<\delta$ and $\left\|\rho_{0}\left(c_{j}\right)-a_{j}\right\|<\delta$ for $j=1,2, \ldots, n$. By the choice of $\delta$, there are a projection $q \in \rho_{0}\left(B_{0}\right)$ and a unitary $u \in A$ satisfying (1).

Since $\mathcal{C}$ has approximate quotients, there is an increasing sequence $I_{0} \subset I_{1} \subset \ldots$ of ideals in $A$ such that $\overline{\bigcup_{n=0}^{\infty} I_{n}}=\operatorname{ker}\left(\rho_{0}\right)$, and such that for every $n$ we have $A / I_{n} \in \mathcal{C}$. By semiprojectivity of $\mathbb{C}$ (see Definition 14.1.3 of [21]), there exists $n$ and a projection $f \in B_{0} / I_{n}$ whose image in $A$ is $q$. Set $B=B_{0} / I_{n}$, which is in $\mathcal{C}$, let $\pi: B_{0} \rightarrow B$ be the quotient map, and let $\rho: B \rightarrow A$ be the unital homomorphism such that $\rho_{0}=\rho \circ \pi$. Define $\varphi: f B f \rightarrow p A p$ by $\varphi(b)=u \rho(b) u^{*}$. Note that $\varphi(b)$ really is in $p A p$, because $u \rho(f) u^{*}=u q u^{*}=p$. Set $b_{j}=f \pi\left(c_{j}\right) f$ for $j=1,2, \ldots, n$. Then

$$
\begin{aligned}
\left\|\varphi\left(b_{j}\right)-a_{j}\right\| & =\left\|u q \rho_{0}\left(c_{j}\right) q u^{*}-a_{j}\right\|=\left\|p u \rho_{0}\left(c_{j}\right) u^{*} p-a_{j}\right\| \leq\left\|u \rho_{0}\left(c_{j}\right) u^{*}-a_{j}\right\| \\
& \leq 2\|u-1\| \cdot\left\|\rho_{0}\left(c_{j}\right)\right\|+\left\|\rho_{0}\left(c_{j}\right)-a_{j}\right\| \\
& <2\left(\frac{\varepsilon}{2 M+3}\right)(M+\delta)+\delta \leq 2\left(\frac{\varepsilon}{2 M+3}\right)(M+1)+\frac{\varepsilon}{2 M+3}=\varepsilon .
\end{aligned}
$$

This shows that $p A p \in \mathcal{L}$.

For the last part, we now know that the hypotheses imply that $\mathcal{L}$ is finitely saturated. Apply Part (4).

Obviously, every unital approximate $\mathcal{C}$-algebra is a unital local $\mathcal{C}$-algebra. We will prove that if $\mathcal{C}$ is flexible, then every local $\mathcal{C}$-algebra is an approximate $\mathcal{C}$ algebra. This is related to, but not quite the same as, the results of Section 15.2 of [21]. The main difference is the issue of injectivity of the maps involved.

In a number of specific cases, this result is known, or is at least implicit in the literature. For example, if $\mathcal{C}=\left\{\mathbb{C}, C([0,1]), C\left(S^{1}\right)\right\}$ (see Example 2.3 below), it is essentially Theorem 4.1 .5 of [19]. There is a slight difference because the definition of a circle algebra used there (Definition 2.3.1 of [19]) allows algebras such as the continuous functions on the Cantor set. Lemma 4.1.2 of 19 implies that every unital AT algebra as defined there is a unital local $\mathcal{C}$-algebra in our sense.

Proposition 1.7. Let $\mathcal{C}$ be a flexible class of separable unital $\mathrm{C}^{*}$-algebras. Then every unital local $\mathcal{C}$-algebra (Definition 1.5) is a unital approximate $\mathcal{C}$-algebra (Definition [1.4).

Proof. By Lemma 1.3. without loss of generality we may assume $\mathcal{C}$ is finitely saturated.

Let $\left\{x_{0}, x_{1}, \ldots\right\} \subset A$ be a countable dense set.

For the purposes of this proof, if $A$ is a unital $\mathrm{C}^{*}$-algebra, $G \subset A$, and $n \in \mathbb{N} \cup\{0\}$, we define $S_{n}(G)$ to be the set of all noncommuting polynomials in the elements $x, x^{*}$ for $x \in G$, with coefficients in $\mathbb{Z}\left[2^{-n}\right]+i \mathbb{Z}\left[2^{-n}\right]$ and of absolute value at most $2^{n}$. Then $S_{n}(G) \subset S_{n+1}(G)$. If $G$ is finite, then so is $S_{n}(G)$. Also, if $G$ generates $A$, then $\bigcup_{n=0}^{\infty} S_{n}(G)$ is dense in $A$. 
We also need finite presentations of our algebras, in the sense of Definition 13.2.2 of [21. We may always require finite generating sets to be bounded by 1 . Thus, following Definition 13.2.1 of [21, for a finite set $G$ of generators we consider the universal unital $\mathrm{C}^{*}$-algebra $F$ freely generated by the family $\left(z_{g}\right)_{g \in G}$ with $\left\|z_{g}\right\| \leq 2$ for each $g \in G$. (In 21, the algebra $F$ is not required to be unital, but all our $\mathrm{C}^{*}$-algebras are unital, so no harm is done by requiring it.) A set $R$ of relations is simply a subset of $F$ such that, if $I \subset F$ is the ideal generated by $R$, then $\left\|z_{g}+I\right\| \leq 1$ for all $g \in G$. The universal unital $\mathrm{C}^{*}$-algebra $C^{*}(G, R)$ is $F / I$.

By Lemma 2.2.5 of [12] (stated in a slightly different setup, but the difference is unimportant), we may always take $R$ to be finite. When $G$ and $R$ are finite, we say that $(G, R)$ is a finite presentation, and that $C^{*}(G, R)$ is finitely presented. See Definition 13.2 .2 of [21].

We construct, by induction on $n$, unital $\mathrm{C}^{*}$-algebras $B_{n} \in \mathcal{C}$ given by finite presentations $B_{n} \cong C^{*}\left(G_{n}, R_{n}\right)$, unital homomorphisms $\psi_{m, n}: B_{m} \rightarrow B_{n}$ for $m \leq n$, positive integers $r(n)$, and unital homomorphisms $\beta_{n}: B_{n} \rightarrow A$, with the following properties:

(1) $\psi_{m, n} \circ \psi_{l, m}=\psi_{l, n}$ for $l \leq m \leq n$.

(2) $\left\|\beta_{n} \circ \psi_{m, n}(b)-\beta_{n-1} \circ \psi_{m, n-1}(b)\right\|<2^{-n}$ for $m \leq n-1$ and $b \in S_{r(n-1)}\left(G_{m}\right)$.

(3) $\left\|\psi_{n-1, n}(b)\right\|<\left\|\beta_{n-1}(b)\right\|+2^{-n+1}$ for $b \in S_{r(n-1)}\left(G_{n-1}\right)$.

(4) $\operatorname{dist}\left(x_{k}, \beta_{n}\left(S_{r(n)}\left(G_{n}\right)\right)\right)<2^{-n}$ for $0 \leq k \leq n$.

(5) $\operatorname{dist}\left(\psi_{m, n}(b), S_{r(n)}\left(G_{n}\right)\right)<2^{-n}$ for $m<n$ and $b \in S_{r(n-1)}\left(G_{m}\right)$.

(6) $r(0)<r(1)<r(2)<\cdots$

Suppose the objects above have been constructed. Let $B=\lim B_{n}$, with homomorphisms $\psi_{n}: B_{n} \rightarrow B$. If $b \in S_{r(n)}\left(G_{m}\right)$ and $n \geq m$, then $k \geq n$ implies

$$
\left\|\beta_{k+1} \circ \psi_{m, k+1}(b)-\beta_{k} \circ \psi_{m, k}(b)\right\|<2^{-k-1}
$$

by Condition (21), so $\lim _{k \rightarrow \infty} \beta_{k} \circ \psi_{m, k}(b)$ exists. Since $\bigcup_{n>m} S_{r(n)}\left(G_{m}\right)$ is dense in $B_{m}$, it follows that $\varphi_{m}(b)=\lim _{k \rightarrow \infty} \beta_{k} \circ \psi_{m, k}(b)$ exists for all $b \in B_{m}$, and defines a unital homomorphism $\varphi_{m}: B_{m} \rightarrow A$ such that $b \in S_{r(m)}\left(G_{m}\right)$ implies $\left\|\varphi_{m}(b)-\beta_{m}(b)\right\|<2^{-m}$. Moreover, $\varphi_{n} \circ \psi_{m, n}=\varphi_{m}$ whenever $m \leq n$, so we get a homomorphism $\varphi: B \rightarrow A$ such that $\varphi \circ \psi_{m}=\varphi_{m}$ for all $m$.

We prove that $\varphi$ is injective. It suffices to prove that if $n \geq m$ and $b \in$ $S_{r(n-1)}\left(G_{m}\right)$, then $\left\|\varphi \circ \psi_{m}(b)\right\|>\left\|\psi_{m}(b)\right\|-2^{-n+2}$. By Condition (5), there is $c \in S_{r(n)}\left(G_{n}\right) \subset B_{n}$ such that $\left\|\psi_{m, n}(b)-c\right\|<2^{-n}$. We have seen that $\left\|\varphi_{n}(c)-\beta_{n}(c)\right\|<2^{-n}$. Therefore, using Condition (3) at the fourth step, we get

$$
\begin{aligned}
\left\|\psi_{m}(b)\right\| & -\left\|\varphi \circ \psi_{m}(b)\right\| \\
& =\left\|\psi_{n} \circ \psi_{m, n}(b)\right\|-\left\|\varphi_{n} \circ \psi_{m, n}(b)\right\| \\
& \leq\left\|\psi_{n}(c)\right\|-\left\|\varphi_{n}(c)\right\|+2\left\|c-\psi_{m, n}(b)\right\| \\
& \leq\left\|\psi_{n, n+1}(c)\right\|-\left\|\beta_{n}(c)\right\|+\left\|\varphi_{n}(c)-\beta_{n}(c)\right\|+2\left\|c-\psi_{m, n}(b)\right\| \\
& <2^{-n}+2^{-n}+2 \cdot 2^{-n}=2^{-n+2} .
\end{aligned}
$$

This completes the proof of injectivity.

For surjectivity, we prove that $\operatorname{dist}\left(x_{m}, \varphi(B)\right)<2^{-n+1}$ for any $n \geq m$. Use Condition (4) to choose $b \in S_{r(n)}\left(G_{n}\right)$ such that $\left\|\beta_{n}(b)-x_{m}\right\|<2^{-n}$. Then $\psi_{n}(b) \in$ 
$B$ and

$$
\begin{aligned}
\left\|\varphi\left(\psi_{n}(b)\right)-x_{m}\right\| & =\left\|\varphi_{n}(b)-x_{m}\right\| \\
& \leq\left\|\varphi_{n}(b)-\beta_{n}(b)\right\|+\left\|\beta_{n}(b)-x_{m}\right\|<2^{-n}+2^{-n}=2^{-n+1},
\end{aligned}
$$

as desired.

It follows that $A \cong \lim _{\longrightarrow} B_{n}$, and is thus a unital approximate $\mathcal{C}$-algebra.

We now carry out the construction.

To start the induction, we use the hypothesis to find a $\mathrm{C}^{*}$-algebra $B_{0} \in \mathcal{C}$ and a unital homomorphism $\beta_{0}: B_{0} \rightarrow A$ such that $\operatorname{dist}\left(x_{0}, \beta_{0}\left(B_{0}\right)\right)<1$. Since $\mathcal{C}$ is flexible, and using Lemma 2.2.5 of [12] as described above, the algebra $B_{0}$ has a finite presentation $B_{0} \cong C^{*}\left(G_{0}, R_{0}\right)$. Since $\bigcup_{r=0}^{\infty} S_{r}\left(G_{0}\right)$ is dense in $B_{0}$, there exists $r(0)$ such that $\operatorname{dist}\left(x_{0}, \beta_{0}\left(S_{r(0)}\left(G_{0}\right)\right)\right)<1$.

Now suppose all objects have been constructed through stage $n$ of the induction.

Since $G_{n}$ generates $B_{n}$, there is $\delta_{0}>0$ such that whenever $D$ is a unital C*algebra and $\mu_{1}, \mu_{2}: B_{n} \rightarrow D$ are unital homomorphisms such that $\left\|\mu_{1}(g)-\mu_{2}(g)\right\|<$ $\delta_{0}$ for all $g \in G_{n}$, then $\left\|\mu_{1}(b)-\mu_{2}(b)\right\|<2^{-n}$ for all $b \in \bigcup_{m \leq n} \psi_{m, n}\left(S_{r(n)}\left(G_{m}\right)\right)$.

Using the notation in the discussion at the beginning of the proof, recall (Definition 13.2.2 of [21]) that a $\delta$-representation of $\left(G_{n}, R_{n}\right)$ in a $\mathrm{C}^{*}$-algebra $D$ is family $\left(a_{g}\right)_{g \in G_{n}}$ in $D$ such that $\left\|a_{g}\right\| \leq 2$ for all $g \in G_{n}$, and the homomorphism $\kappa: F \rightarrow D$ such that $\kappa\left(z_{g}\right)=a_{g}$ for all $g \in G_{n}$ satisfies $\|\kappa(b)\| \leq \delta$ for all $b \in R$. Since $B_{n} \in \mathcal{C}$, it is semiprojective, and Theorem 14.1.4 of [21] implies that the set $R_{n}$ of relations is stable. Therefore there exists $\delta_{1}>0$ such that for every $\delta_{1}$-representation $\left(a_{g}\right)_{g \in G_{n}}$ of $\left(G_{n}, R_{n}\right)$ in a $\mathrm{C}^{*}$-algebra $D$, there is a representation $\left(b_{g}\right)_{g \in G_{n}}$ of $\left(G_{n}, R_{n}\right)$ in $D$ such that $\left\|b_{g}-a_{g}\right\|<\frac{1}{2} \delta_{0}$ for all $g \in G_{n}$. Then use finiteness of $R_{n}$ to choose $\delta_{2}>0$ such that $\delta_{2} \leq \min \left(2^{-n-1}, \frac{1}{2} \delta_{1}, \delta_{0}\right)$ and so small that whenever $\left(c_{g}\right)_{g \in G_{n}}$ is a representation of the relations $R_{n}$ in a $\mathrm{C}^{*}$-algebra $D$, and $\left\|c_{g}-d_{g}\right\|<\delta_{2}$ for all $g \in G$, then $\left(d_{g}\right)_{g \in G}$ is a $\frac{1}{2} \delta_{1}$-representation of $\left(G_{n}, R_{n}\right)$.

Define $T=\left\{x_{0}, \ldots, x_{n+1}\right\} \cup \beta_{n}\left(G_{n}\right)$, which is a finite subset of $A$. By hypothesis, there exists a $C^{*}$-algebra $C \in \mathcal{C}$ and a unital homomorphism $\gamma: C \rightarrow A$ such that $\operatorname{dist}(x, \gamma(C))<\frac{1}{2} \delta_{2}$ for all $x \in T$. For each $g \in G_{n}$ choose $c_{g} \in C$ such that $\left\|\gamma\left(c_{g}\right)-\beta_{n}(g)\right\|<\frac{1}{2} \delta_{2}$. By choice of $\delta_{2}$, the elements $\gamma\left(c_{g}\right)$ define a $\frac{1}{2} \delta_{1}$ representation of $\left(G_{n}, R_{n}\right)$. (Note that $\left\|\gamma\left(c_{g}\right)\right\|<\left\|\beta_{n}(g)\right\|+\frac{1}{2} \delta_{2} \leq 1+\frac{1}{2} \delta_{2}<2$ for $g \in G_{n}$.) Let $\kappa: F \rightarrow A$ be the associated homomorphism, so $\|\kappa(d)\| \leq \frac{1}{2} \delta_{1}$ for all $d \in R_{n}$.

By Condition (11) of Definition 1.2, there exists an increasing sequence $I_{0} \subset$ $I_{1} \subset \cdots$ of ideals in $C$ such that $\overline{\bigcup_{l=0}^{\infty} I_{l}}=\operatorname{Ker}(\gamma)$ and $C / I_{l} \in \mathcal{C}$ for every $l$. Let $\gamma=\gamma_{l} \circ \pi_{l}$ be the factorization of $\gamma$ through $C / I_{l}$. Then there exists $l_{0}$ such that $\left\|\pi_{l}\left(c_{g}\right)\right\|<2$ for all $l \geq l_{0}$ and $g \in G_{n}$. Thus, for all $l \geq l_{0}$ there is a homomorphism $\kappa_{l}: F \rightarrow C / I_{l}$ such that $\kappa_{l}\left(z_{g}\right)=\pi_{l}\left(c_{g}\right)$ for $g \in G_{n}$, and $\gamma_{l} \circ \kappa_{l}=\kappa$. Since $R_{n}$ is finite, we may choose $l_{1} \geq l_{0}$ so large that $l \geq l_{1}$ implies $\left\|\kappa_{l}(d)\right\| \leq\|\kappa(d)\|+\frac{1}{2} \delta_{1} \leq \delta_{1}$ for all $d \in R_{n}$. Then $\kappa_{l}$ is a $\delta_{1}$-representation of $\left(G_{n}, R_{n}\right)$. By the choice of $\delta_{1}$, there is therefore a homomorphism $\lambda: B_{n} \rightarrow C / I_{l_{1}}$ such that $\left\|\lambda(g)-\kappa_{l}\left(z_{g}\right)\right\|<\frac{1}{2} \delta_{0}$ for all $g \in G_{n}$. Then for $g \in G_{n}$, using $\kappa_{l_{1}}\left(z_{g}\right)=\pi_{l_{1}}\left(c_{g}\right)$, we have

$$
\left\|\gamma_{l_{1}}(\lambda(g))-\beta_{n}(g)\right\| \leq\left\|\lambda(g)-\kappa_{l_{1}}\left(z_{g}\right)\right\|+\left\|\gamma\left(c_{g}\right)-\beta_{n}(g)\right\|<\frac{1}{2} \delta_{0}+\frac{1}{2} \delta_{2} \leq \delta_{0} .
$$

Now $\left\|\gamma_{l_{1}}(\lambda(b))-\beta_{n}(b)\right\|<2^{-n}$ for all $b \in \bigcup_{m \leq n} \psi_{m, n}\left(S_{r(n)}\left(G_{m}\right)\right)$ by the choice of $\delta_{0}$. 
For $l \geq l_{1}$, let $\mu_{l}$ be the quotient map $\mu_{l}: C / I_{l_{1}} \rightarrow C / I_{l}$. Then for every $b \in$ $S_{r(n)}\left(G_{n}\right)$, we have

$$
\lim _{l \rightarrow \infty}\left\|\mu_{l}(\lambda(b))\right\|=\left\|\gamma_{l_{1}}(\lambda(b))\right\|<\left\|\beta_{n}(b)\right\|+2^{-n} .
$$

Therefore there exists $l_{2} \geq l_{1}$ such that $\left\|\mu_{l_{2}}(\lambda(b))\right\|<\left\|\beta_{n}(b)\right\|+2^{-n}$ for all $b \in$ $S_{r(n)}\left(G_{n}\right)$.

We now define $B_{n+1}=C / I_{l_{2}}, \beta_{n+1}=\gamma_{l_{2}}$, and $\psi_{n, n+1}=\mu_{l_{2}} \circ \lambda$. For $m<n$ set $\psi_{m, n+1}=\psi_{n, n+1} \circ \psi_{m, n}$. Condition (11) is immediate. Condition (2) follows from the equation $\gamma_{l_{1}} \circ \lambda=\gamma_{l_{2}} \circ \mu_{l_{2}} \circ \lambda$ and the estimate, proved in slightly different notation above, $\left\|\gamma_{l_{1}} \circ \lambda \circ \psi_{m, n}(b)-\beta_{n} \circ \psi_{m, n}(b)\right\|<2^{-n}$ for $m \leq n$ and $b \in$ $S_{r(n)}\left(G_{m}\right)$. Condition (3) has just been proved. Since $B_{n+1} \in \mathcal{C}$, there is a finite presentation $B_{n+1} \cong C^{*}\left(G_{n+1}, R_{n+1}\right)$ in the sense of Definition 13.2.2 of [21. Then $\bigcup_{r=0}^{\infty} S_{r}\left(G_{n+1}\right)$ is dense in $B_{n+1}$. For $k \leq n+1$, we have

$$
\operatorname{dist}\left(x_{k}, \beta_{n+1}\left(B_{n+1}\right)\right)=\operatorname{dist}\left(x_{k}, \gamma(C)\right)<\delta_{2} \leq 2^{-n-1},
$$

so it is easy to choose $r(n+1)>r(n)$ and also so large that Conditions (4) and (5D) are satisfied. This completes the induction, and thus the proof of the proposition.

\section{EXAMPLES OF FLEXiBle ClASSES}

In this section, we prove that many of the popular classes of semiprojective building blocks are flexible.

Example 2.1. Let $\mathcal{C}=\{\mathbb{C}\}$. Then $\mathcal{C}$ is flexible. The finite saturation of $\mathcal{C}$ consists of all finite dimensional $\mathrm{C}^{*}$-algebras. The unital approximate $\mathcal{C}$-algebras are exactly the unital AF algebras.

Example 2.2. Let $\mathcal{C}=\{\mathbb{C}, C([0,1])\}$. We claim that $\mathcal{C}$ is flexible.

We verify Condition (2) of Definition 1.2 Both $\mathbb{C}$ and $C([0,1])$ are well known to be finitely generated and semiprojective. Thus, for any $k$, the algebras $M_{k}(\mathbb{C})$ and $M_{k}(C([0,1]))$ are finitely generated (easy) and semiprojective (Theorem 14.2.1 of 21]). Now suppose $A \in \mathcal{C}$ and $p \in M_{n}(A)$ is a projection. Then there is $k$ such that $p M_{n}(A) p \cong M_{k}(A)$, which we just dealt with.

Now we verify Condition (11) of Definition 1.2, For $A=\mathbb{C}$ this condition is trivial. So let $I \subset C([0,1])$ be an ideal. Write

$$
I=\left\{f \in C([0,1]):\left.f\right|_{Y}=0\right\}
$$

for a closed set $Y \subset[0,1]$. Since $[0,1] \backslash Y$ is the disjoint union of at most countably many open intervals, we can write $Y=\bigcap_{n=0}^{\infty} Y_{n}$ for closed sets $Y_{0} \supset Y_{1} \supset \cdots$ such that each $[0,1] \backslash Y_{n}$ is a finite union of open intervals. Take

$$
I_{n}=\left\{f \in C([0,1]):\left.f\right|_{Y_{n}}=0\right\} .
$$

Then $C([0,1]) / I_{n} \cong C\left(Y_{n}\right)$ is a finite direct sum of $\mathrm{C}^{*}$-algebras isomorphic to $\mathbb{C}$ or $C([0,1])$. This completes the proof that $\mathcal{C}$ is flexible.

The unital approximate $\mathcal{C}$-algebras are the unital AI algebras. Since we have seen several definitions of an AI algebra, we formally take this as the definition in this paper.

Example 2.3. Let $\mathcal{C}=\left\{\mathbb{C}, C([0,1]), C\left(S^{1}\right)\right\}$. Then $\mathcal{C}$ is flexible by the same argument as in Example 2.2. The unital approximate $\mathcal{C}$-algebras are the unital AT algebras. Again, we take this as the definition of a unital AT algebra. 
Example 2.4. For $n \in \mathbb{N}$, let $D_{n}$ be the dimension drop interval,

$$
D_{n}=\left\{f \in C\left([0,1], M_{n}\right): f(0) \text { and } f(1) \text { are scalars }\right\} .
$$

Further set

$$
C_{n}=\left\{f \in C\left([0,1], M_{n}\right): f(0) \text { is a scalar }\right\} .
$$

Let

$$
\mathcal{C}=\{\mathbb{C}, C([0,1])\} \cup\left\{C_{n}, D_{n}: n \geq 2\right\} .
$$

This collection satisfies Condition (2) of Definition 1.2 because it is contained in the class of Example 2.8 below. The argument in Example 2.2 for Condition (11) of Definition 1.2 applies directly, so $\mathcal{C}$ is flexible.

We define a unital AD algebra ("approximate dimension drop algebra") to be a unital approximate $\mathcal{C}$-algebra.

One should note that the collection $\mathcal{C}$ in Example 2.4 would not be flexible without the algebras $C_{n}$, since Condition (1) of Definition 1.2 would fail.

We now consider one dimensional noncommutative $\mathrm{CW}$ complexes. This class requires some preliminary work. We recall Definition 2.4.1 of [12] (which is a special case of Definition 11.2 of [27]):

Definition 2.5. A one dimensional noncommutative $\mathrm{CW}$ complex is a $\mathrm{C}^{*}$-algebra $A$ of the form

$$
A=\left\{(a, f) \in F_{0} \oplus C\left([0,1], F_{1}\right): f(0)=\varphi_{0}(a) \text { and } f(1)=\varphi_{1}(a)\right\},
$$

where $F_{0}$ and $F_{1}$ are finite dimensional $\mathrm{C}^{*}$-algebras and $\varphi_{0}, \varphi_{1}: F_{0} \rightarrow F_{1}$ are unital homomorphisms.

Lemma 2.6. Let the notation be as in Definition 2.5. Write $F_{1}=\bigoplus_{k=1}^{n} M_{d(k)}$, and write $(a, f) \in A$ as $\left(a, f_{1}, f_{2}, \ldots, f_{n}\right)$ with $f_{k} \in C\left([0,1], M_{d(k)}\right)$. For each $k$, let $\alpha_{j, k}$ and $\beta_{j, k}$, for $1 \leq j \leq r(k)$, satisfy

$$
0 \leq \alpha_{1, k}<\beta_{1, k}<\alpha_{2, k}<\beta_{2, k}<\cdots<\alpha_{r(k), k}<\beta_{r(k), k} \leq 1 .
$$

Let $J \subset A$ be the ideal consisting of all $(a, f) \in A$ such that $\left.f_{k}\right|_{\left[\alpha_{j, k}, \beta_{j, k}\right]}=0$ for $1 \leq k \leq n$ and $1 \leq j \leq r(k)$. Then $A / J$ is a one dimensional noncommutative $\mathrm{CW}$ complex of the form

$$
A / J=\left\{(b, g) \in G_{0} \oplus C\left([0,1], G_{1}\right): g(0)=\psi_{0}(b) \text { and } g(1)=\psi_{1}(b)\right\},
$$

with $G_{0}$ and $G_{1}$ finite dimensional and $\psi_{0}, \psi_{1}: G_{0} \rightarrow G_{1}$ unital. Moreover, there is an expression of this type such that if $(a, f) \in A$ and $(a, f)+J=(b, g)$, then

$$
\|g\| \leq \max _{1 \leq k \leq n} \max _{1 \leq j \leq r(k)}\left\|\left.f_{k}\right|_{\left[\alpha_{j, k}, \beta_{j, k}\right]}\right\| .
$$

Proof. Let $\pi: A \rightarrow A / J$ be the quotient map.

Define $G_{1}^{(k)}=M_{d(k)}^{r(k)}$, the direct sum of $r(k)$ copies of $M_{d(k)}$. Define $G_{1}=$ $\bigoplus_{k=1}^{n} G_{1}^{(k)}$. If $\alpha_{1, k}=0$ set $G_{0}^{(0, k)}=M_{d(k)}^{r(k)-1}$; otherwise set $G_{0}^{(0, k)}=G_{1}^{(k)}$. Similarly, set $G_{0}^{(1, k)}=M_{d(k)}^{r(k)-1}$ if $\beta_{r(k), k}=1$ and set $G_{0}^{(1, k)}=G_{1}^{(k)}$ otherwise. Further set

$$
G_{0}=F_{0} \oplus \bigoplus_{k=1}^{n}\left(G_{0}^{(0, k)} \oplus G_{0}^{(1, k)}\right) .
$$

Define $\psi_{0, k}: G_{0} \rightarrow G_{1}^{(k)}$ as follows. Let

$$
x=\left(a, b_{0,1}, b_{1,1}, b_{0,2}, b_{1,2}, \ldots, b_{0, n}, b_{1, n}\right) \in G_{0} .
$$


Write $\varphi_{0}(a)_{k}$ for the $k$ th coordinate of $\varphi_{0}(a) \in \bigoplus_{k=1}^{n} M_{d(k)}$. Then set

$$
\psi_{0, k}(x)= \begin{cases}\left(\varphi_{0}(a)_{k}, b_{0, k}\right) & \alpha_{1, k}=0 \\ b_{0, k} & \alpha_{1, k}>0\end{cases}
$$

and

$$
\psi_{1, k}(x)= \begin{cases}\left(b_{0, k}, \varphi_{1}(a)_{k}\right) & \beta_{1, k}=1 \\ b_{0, k} & \beta_{1, k}<1 .\end{cases}
$$

Combine these in the obvious way to make unital homomorphisms $\psi_{0}, \psi_{1}: G_{0} \rightarrow$ $G_{1}$. Set

$$
B=\left\{(b, g) \in G_{0} \oplus C\left([0,1], G_{1}\right): g(0)=\psi_{0}(b) \text { and } g(1)=\psi_{1}(b)\right\} .
$$

We show that $A / J \cong B$. Define $\rho_{0}: A \rightarrow G_{0}$ by

$$
\rho_{0}(a, f)=\left(a, b_{0,1}, b_{1,1}, b_{0,2}, b_{1,2}, \ldots, b_{0, n}, b_{1, n}\right) \text {, }
$$

with

$$
b_{0, k}= \begin{cases}\left(f_{k}\left(\alpha_{j, k}\right)\right)_{2 \leq j \leq r(k)} & \alpha_{1, k}=0 \\ \left(f_{k}\left(\alpha_{j, k}\right)\right)_{1 \leq j \leq r(k)} & \alpha_{1, k}>0\end{cases}
$$

and

$$
b_{1, k}= \begin{cases}\left(f_{k}\left(\beta_{j, k}\right)\right)_{1 \leq j \leq r(k)-1} & \beta_{1, k}=1 \\ \left(f_{k}\left(\beta_{j, k}\right)\right)_{1 \leq j \leq r(k)} & \beta_{1, k}<1 .\end{cases}
$$

For each $j$ and $k$, choose an orientation preserving homeomorphism $h_{j, k}:[0,1] \rightarrow$ $\left[\alpha_{j, k}, \beta_{j, k}\right]$. Then define $\rho_{1}: A \rightarrow C\left([0,1], G_{1}\right)$ by

$$
\rho_{1}(a, f)=\left(\left(f_{1} \circ h_{j, 1}\right)_{1 \leq j \leq r(1)},\left(f_{2} \circ h_{j, 2}\right)_{1 \leq j \leq r(2)}, \ldots,\left(f_{n} \circ h_{j, n}\right)_{1 \leq j \leq r(n)}\right),
$$

and define $\rho: A \rightarrow B$ by $\rho(a, f)=\left(\rho_{0}(a, f), \rho_{1}(a, f)\right)$. One checks easily that $\rho(a, f)$ really is in $B$ and that $\rho(a, f)=0$ if and only if $f \in J$. It remains to show that $\rho$ is surjective. Let $(b, g) \in B$. Let $a$ be the projection of $b$ to the summand $F_{0}$ of $G_{0}$. Let $1 \leq k \leq n$. Set

$$
L_{k}=\{0,1\} \cup \bigcup_{j=1}^{r(k)}\left[\alpha_{j, k}, \beta_{j, k}\right] .
$$

We claim that there exists a unique function $f_{k}^{(0)} \in C\left(L_{k}, M_{d(k)}\right)$ such that $f_{k}^{(0)}(0)=$ $\varphi_{0}(a)_{k}, f_{k}^{(0)}(1)=\varphi_{1}(a)_{k}$, and the functions $f_{k}^{(0)} \circ h_{j, k}$ are equal to the corresponding components of $g$. This is immediate unless $\alpha_{1, k}=0$ or $\beta_{r(k), k}=1$, in which case $f_{k}^{(0)}(0)$ or $f_{k}^{(0)}(1)$ must satisfy two conditions. In these cases, however, the requirements $g(0)=\psi_{0}(b)$ and $g(1)=\psi_{1}(b)$ ensure that the two conditions agree. Now extend $f_{k}^{(0)}$ to a continuous function $f_{k} \in C\left([0,1], M_{d(k)}\right)$, and observe that $\left(a, f_{1}, f_{2}, \ldots, f_{n}\right) \in A$ and satisfies $\rho\left(a, f_{1}, f_{2}, \ldots, f_{n}\right)=(b, g)$. This completes the proof, except for the norm estimate, which is clear from the construction.

Lemma 2.7. Let $A$ be a one dimensional noncommutative $\mathrm{CW}$ complex, and let $I$ be an ideal in $A$. Then there exists an increasing sequence $I_{0} \subset I_{1} \subset \cdots$ of ideals in $A$ such that $\overline{\bigcup_{n=0}^{\infty} I_{n}}=I$, and such that for every $n$ the $\mathrm{C}^{*}$-algebra $A / I_{n}$ is a one dimensional noncommutative $\mathrm{CW}$ complex. 
Proof. Let the notation be as in Definition 2.5. We claim that it suffices to consider the case in which $\left(\varphi_{0}, \varphi_{1}\right): F_{0} \rightarrow F_{1} \oplus F_{1}$ is injective. If this map is not injective, let $L$ be its kernel, and write $A=L \oplus B$ where $B$ is the one dimensional noncommutative CW complex defined using $F_{0} / L$ and the induced maps $F_{0} / L \rightarrow F_{1}$ in place of $F_{0}, \varphi_{0}$, and $\varphi_{1}$. It suffices to consider the summands separately, so the claim follows.

We first claim that, under the hypotheses of the lemma, and assuming the injectivity condition of the previous paragraph, if $x \in I$ then for every $\varepsilon>0$ there is an ideal $J \subset I$ such that $A / J$ is a one dimensional noncommutative CW complex satisfying the injectivity condition of the previous paragraph and such that $\|x+J\|<\varepsilon$. Write $F_{1}=\bigoplus_{k=1}^{n} M_{d(k)}$, and write $x=\left(a, f_{1}, f_{2}, \ldots, f_{n}\right)$ with $a \in F_{0}$ and $f_{k} \in C\left([0,1], M_{d(k)}\right)$. Let

$$
U_{k}=\left\{t \in[0,1]:\left\|f_{k}(t)\right\|>0\right\} \quad \text { and } \quad L_{k}=\left\{t \in[0,1]:\left\|f_{k}(t)\right\| \geq \frac{1}{2} \varepsilon\right\} .
$$

Since $U_{k}$ is a countable disjoint union of open intervals in $[0,1]$ (we include intervals of the form $[0, s)$ and $(s, 1])$, we may write $U_{k}=\bigcup_{m=1}^{\infty} V_{m, k}$, where each $V_{m, k}$ is a finite union of open intervals, the closures of which are contained in distinct intervals in the expression of $U_{k}$ as a disjoint union of open intervals. By compactness, one of these sets, call it $W_{k}$, contains $L_{k}$. Let $J_{0}$ be the ideal in $A$ consisting of all $\left(b, g_{1}, g_{2}, \ldots, g_{n}\right) \in A$ such that $\left.g_{k}\right|_{[0,1] \backslash W_{k}}=0$ for $1 \leq k \leq n$. The sets $[0,1] \backslash W_{k}$ have the form in the hypotheses of Lemma 2.6. so $A / J_{0}$ is a one dimensional noncommutative $\mathrm{CW}$ complex of the form

$$
A / J_{0}=\left\{\left(b_{0}, g\right) \in G_{0} \oplus C\left([0,1], G_{1}\right): g(0)=\psi_{0}(b) \text { and } g(1)=\psi_{1}(b)\right\},
$$

such that if we write $x+J_{0}=(b, g)$, then $\|g\|<\varepsilon$. Set $M=\operatorname{Ker}\left(\psi_{0}\right) \cap \operatorname{Ker}\left(\psi_{1}\right)$. Define a new one dimensional noncommutative CW complex $B$ by replacing $G_{0}$ with $G_{0} / M$, and making the obvious replacements for $\psi_{0}$ and $\psi_{1}$. The map $G_{0} / M \rightarrow$ $G_{1} \oplus G_{1}$ they induce is injective. Thus, letting $J$ be the kernel of $A \rightarrow B$, we have $\|x+J\| \leq\|g\|<\varepsilon$. This completes the proof of the claim.

Now we prove the lemma. Let $\left(x_{n}\right)_{n \in \mathbb{N}}$ be a sequence in $I$ such that every tail $\left(x_{n}\right)_{n \geq N}$ is dense. We apply the claim to $I \subset A$ with $x_{1}$ in place of $x$ and with $\varepsilon=1$, obtaining an ideal $I_{1} \subset I$. Let $\pi_{1}: A \rightarrow A / I_{1}$ be the quotient map. Now apply the claim to $I / I_{1} \subset A / I_{1}$ with $\pi_{1}\left(x_{2}\right)$ in place of $x$ and with $\varepsilon=\frac{1}{2}$, obtaining an ideal $J_{2} \subset I / I_{1}$. Take $I_{2}=\pi_{1}^{-1}\left(J_{2}\right)$, and let $\pi_{2}: A \rightarrow A / I_{2}$ be the quotient map. Then apply the claim to $I / I_{2} \subset A / I_{2}$ with $\pi_{2}\left(x_{3}\right)$ in place of $x$ and with $\varepsilon=\frac{1}{3}$, obtaining an ideal $J_{3} \subset I / I_{2}$, and take $I_{3}=\pi_{2}^{-1}\left(J_{3}\right)$, etc. For every $N$, every element of the dense sequence $\left(x_{n}\right)_{n \geq N}$ in $I$ can be approximated within $1 / N$ by elements of $\bigcup_{n=1}^{\infty} I_{n}$, so that $\bigcup_{n=1}^{\infty} I_{n}$ is dense in $I$.

Example 2.8. Let $\mathcal{C}$ be the collection of all $\mathrm{C}^{*}$-algebras isomorphic to one dimensional noncommutative $\mathrm{CW}$ complexes as in Definition 2.5. We claim that $\mathcal{C}$ is flexible. One easily checks $\mathcal{C}$ is finitely saturated. Algebras in $\mathcal{C}$ are finitely generated by Lemma 2.4.3 of [12, and semiprojective by Theorem 6.2.2 of [12]. Thus, Condition (2) of Definition 1.2 is satisfied. Condition (11) of Definition 1.2 follows from Lemma 2.7

Example 2.9. For $d \in \mathbb{N}$, take $\mathcal{C}_{d}$ to be the collection of all $\mathrm{C}^{*}$-algebras $C(X)$ for $X$ a compact metric space with covering dimension at most $d$. Then a separable unital $\mathrm{C}^{*}$-algebra $A$ is an $\mathrm{AH}$ algebra with no dimension growth if and only if it is a unital approximate $\mathcal{C}_{d^{-}}$algebra for some $d$. 
The class $\mathcal{C}_{d}$ is not flexible. (For $d=0$, it contains the algebra of continuous functions on the Cantor set, which is not semiprojective.) Even if one restricts to finite complexes, one will not get a flexible class for $d \geq 2$.

Example 2.10. Let $T$ be the Toeplitz algebra, the $\mathrm{C}^{*}$-algebra of the unilateral shift. Then the class $\left\{\mathbb{C}, C([0,1]), C\left(S^{1}\right), T\right\}$ is flexible. The verification of Condition (1) of Definition 1.2 for $T$ reduces to that for $C\left(S^{1}\right)$. For Condition (2), let $p \in M_{n}(T)$ be a nonzero projection. Let $\pi: M_{n}(T) \rightarrow M_{n}\left(C\left(S^{1}\right)\right)$ be the quotient map. If $\pi(p)=0$, then $p M_{n}(T) p$ is finite dimensional, hence obviously finitely generated and projective. Otherwise, there is a short exact sequence

$$
0 \longrightarrow p K p \longrightarrow p M_{n}(T) p \longrightarrow \pi(p) M_{n}\left(C\left(S^{1}\right)\right) \pi(p) \longrightarrow 0 .
$$

Clearly $\pi(p)$ is full, so $p$ is full. Now $T$ is semiprojective by Corollary 2.22 of [2], so $M_{n}(T)$ is semiprojective by Theorem 14.2.2 of [21], whence $p M_{n}(T) p$ is semiprojective by Proposition 2.27 of [2]. Moreover, $\pi(p) M_{n}\left(C\left(S^{1}\right)\right) \pi(p)$ is finitely generated as in Example 2.3. and $p K p \cong K$ is finitely generated by Theorem 8 of [23], so $p M_{n}(T) p$ is finitely generated.

We now consider examples using purely infinite simple $\mathrm{C}^{*}$-algebras. The following lemma is useful. The conclusion can be improved to "singly generated"; see Example (4) on page 138 of [22]. However, no proof is given there, and the version here is easy to prove and suffices for our purposes.

Lemma 2.11. Every separable unital purely infinite simple $\mathrm{C}^{*}$-algebra is finitely generated.

Proof. Let $A$ be a separable unital purely infinite simple $\mathrm{C}^{*}$-algebra. Choose nonzero projections $p_{1}, p_{2}, p_{3} \in A$ such that $p_{1}+p_{2}+p_{3}=1$. There exist mutually orthogonal projections $e_{1}, e_{2}, \ldots \leq p_{1}$, each equivalent to $1-p_{1}$, and the hereditary subalgebra $B$ of $A$ generated by $\left\{1-p_{1}\right\} \cup\left\{e_{1}, e_{2}, \ldots\right\}$ is isomorphic to $K \otimes\left(1-p_{1}\right) A\left(1-p_{1}\right)$. It follows from Theorem 8 of 23 , that $B$ is generated by a single element $a_{1}$. Similarly, there exist $a_{2}, a_{3} \in A$ which generate subalgebras which contain $\left(1-p_{2}\right) A\left(1-p_{2}\right)$ and $\left(1-p_{3}\right) A\left(1-p_{3}\right)$. In particular, $\left\{a_{1}, a_{2}, a_{3}\right\}$ generates a subalgebra $A_{0}$ of $A$ which contains $\left(1-p_{j}\right) A\left(1-p_{j}\right)$ for $j \in\{1,2,3\}$. If $j, k \in\{1,2,3\}$ are given, and $l$ is equal to neither $j$ nor $k$, then $p_{j} A p_{k} \subset\left(1-p_{l}\right) A\left(1-p_{l}\right)$. So $A_{0}$ contains $p_{j} A p_{k}$ for all $j$ and $k$, and hence contains $A$.

Example 2.12. The class of Cuntz algebras $\mathcal{C}=\left\{\mathcal{O}_{m}: 2 \leq m<\infty\right\}$ is flexible. For the proof, $\mathcal{O}_{m}$ is semiprojective by Corollary 2.24 of [2], so $M_{n}\left(\mathcal{O}_{m}\right)$ is semiprojective by Theorem 14.2.2 of 21. Every corner in such an algebra is full, hence semiprojective by Proposition 2.27 of [2]. It follows from Lemma 2.11] that all such corners are finitely generated.

Every (nonempty) subset of $\mathcal{C}$ is also flexible.

Example 2.13. The class $\left\{\mathcal{O}_{\infty}\right\}$ is flexible. Indeed, this algebra is semiprojective by Theorem 3.2 of [5], and the rest of the argument is as in Example 2.12.

Example 2.14. The union of flexible classes is flexible. Thus, for example, the class $\left\{\mathbb{C}, C([0,1]), C\left(S^{1}\right), \mathcal{O}_{2}\right\}$ is flexible, by Examples 2.3 and 2.12 and the class $\left\{\mathcal{O}_{m}: 2 \leq m \leq \infty\right\}$ is flexible, by Examples 2.12 and 2.13 .

Example 2.15. Let $E_{m}$ be the standard extension of $\mathcal{O}_{m}$ by $K$. Then the class $\left\{\mathbb{C}, \mathcal{O}_{m}, E_{m}\right\}$ is flexible. The algebra $\mathcal{O}_{m}$ is semiprojective by Corollary 2.24 of [2]. 
The algebra $E_{m}$ is easily seen to be semiprojective by using Propositions 2.18 and 2.23 of [2]. The proof of Condition (2) of Definition 1.2 is now the same as for Example 2.10, but using semiprojectivity and finite generation of corners and matrix algebras over $\mathcal{O}_{m}$ as in Example 2.12,

\section{Structure theorems For CRossed PRoducts By aCtions With the ROKHLIN PROPERTY}

In this section, we prove various results of the form discussed in the introduction, to the effect that crossed products by finite group actions with the Rokhlin property preserve various properties of $\mathrm{C}^{*}$-algebras. We concentrate in membership in various classes considered in the Elliott program; other properties are considered in the next section. The Rokhlin property is as in Definition 3.1 of [16]; we reproduce here the equivalent form given in Definition 1.1 of [28.

Definition 3.1. Let $A$ be a unital $\mathrm{C}^{*}$-algebra, and let $\alpha: G \rightarrow \operatorname{Aut}(A)$ be an action of a finite group $G$ on $A$. We say that $\alpha$ has the Rokhlin property if for every finite set $F \subset A$ and every $\varepsilon>0$, there are mutually orthogonal projections $e_{g} \in A$ for $g \in G$ such that:

(1) $\left\|\alpha_{g}\left(e_{h}\right)-e_{g h}\right\|<\varepsilon$ for all $g, h \in G$.

(2) $\left\|e_{g} a-a e_{g}\right\|<\varepsilon$ for all $g \in G$ and all $a \in F$.

(3) $\sum_{g \in G} e_{g}=1$.

The proof of the following theorem is contained in the proof of Theorem 2.2 of 28 . We will mostly use the version in Theorem 3.3 below, but one of our results requires this version.

Theorem 3.2. Let $A$ be a unital $\mathrm{C}^{*}$-algebra, let $G$ be a finite group, and let $\alpha: G \rightarrow \operatorname{Aut}(A)$ be an action with the Rokhlin property. Then for every finite subset $S \subset C^{*}(G, A, \alpha)$ and every $\varepsilon>0$, there are $n$, a projection $f \in A$, and a unital homomorphism $\varphi: M_{n} \otimes f A f \rightarrow C^{*}(G, A, \alpha)$ such that $\operatorname{dist}\left(a, \varphi\left(M_{n} \otimes f A f\right)\right)<\varepsilon$ for all $a \in S$.

Proof. This is what is actually shown in the proof of Theorem 2.2 of [28. (We take $f$ to be the projection $e_{1}$ used there.)

Theorem 3.2 has the following consequence, which is usually what is actually wanted. When $A$ is assumed to be an AH algebra with no dimension growth, the conclusion was first obtained by Dawn Archey.

Theorem 3.3. Let $\mathcal{C}$ be any class of separable unital $\mathrm{C}^{*}$-algebras which has approximate quotients (Definition 1.2). Let $A$ be a unital local $\mathcal{C}$-algebra. Let $G$ be a finite group, and let $\alpha: G \rightarrow \operatorname{Aut}(A)$ be an action with the Rokhlin property. Then $C^{*}(G, A, \alpha)$ is again a unital local $\mathcal{C}$-algebra.

Proof. Parts (2) and (5) of Lemma 1.6 show that the algebras $M_{n} \otimes f A f$ in Theorem 3.2 are unital local $\mathcal{C}$-algebras. Therefore Theorem 3.2 and Lemma 1.6(4) imply that $C^{*}(G, A, \alpha)$ is a unital local $\mathcal{C}$-algebra.

In Theorem 3.2, one might hope that one can choose $f$ so that $f A f$ is stably isomorphic to a direct summand of $A$. If so, in Theorem 3.3 one could replace having approximate quotients by a weaker hypothesis. One would obtain, for example, a strengthening of Proposition 3.7 below, in which nothing need be said about 
quotients. Unfortunately, the following example shows that, in general, it is not possible to choose $f$ to satisfy this condition. (The example does not, however, show that the proposed strengthening of Proposition 3.7 is false.)

Example 3.4. Let $B=\bigotimes_{n=1}^{\infty} M_{2}$ be the $2^{\infty}$ UHF algebra. We set $B_{n}=\bigotimes_{k=1}^{n} M_{2}$, and identify $B$ with $\lim _{\longrightarrow} B_{n}$. Let $\beta_{0}=\bigotimes_{n=1}^{\infty} \operatorname{Ad}\left(\left(\begin{array}{cc}1 & 0 \\ 0 & -1\end{array}\right)\right) \in \operatorname{Aut}(B)$. Let $K=$ $K\left(l^{2}(\mathbb{N})\right)$, and for $j, k \in \mathbb{N}$ let $f_{j, k}$ denote the standard matrix unit. Further let $f_{n}=\sum_{k=1}^{2 n} f_{k, k}$ and $g_{n}=\sum_{k=1}^{n} f_{2 k, 2 k}$. In the multiplier algebra $M(K \otimes B)$ define (with convergence in the strict topology)

$$
g=\lim _{n \rightarrow \infty} g_{n} \otimes 1=\sum_{k=1}^{\infty} f_{2 k, 2 k} \otimes 1 \quad \text { and } \quad u=\sum_{k=1}^{\infty}\left(f_{2 k-1,2 k}+f_{2 k, 2 k-1}\right) \otimes 1 .
$$

Then $u$ is unitary, $u^{2}=1$, and $u g u^{*}=1-g$. Define $A \subset M(K \otimes B)$ to be the subalgebra generated by $1, g$, and $K \otimes B$. Let $\beta \in \operatorname{Aut}(M(K \otimes B))$ be the extension to $M(K \otimes B)$ of $\operatorname{id}_{K} \otimes \beta_{0}$, and let $\alpha \in \operatorname{Aut}(A)$ be the restriction to $A$ of $\operatorname{Ad}(u) \circ \beta$. Note that $(\operatorname{Ad}(u) \circ \beta)(A)=A$, so that we really do have $\alpha \in \operatorname{Aut}(A)$. Moreover, since $\operatorname{Ad}(u)$ and $\beta$ are commuting automorphisms of order 2 , we easily see that $\alpha^{2}=\operatorname{id}_{A}$.

We claim that $\alpha$ generates an action of $\mathbb{Z} / 2 \mathbb{Z}$ which has the Rokhlin property. Let $F_{n} \subset A$ be the set

$$
F_{n}=\{1, g\} \cup\left\{e_{j, k} \otimes b: j, k \leq 2 n \text { and } b \in B_{n}\right\} .
$$

The sets $F_{n}$ are not finite, but $F_{1} \subset F_{2} \subset \cdots$ and $\bigcup_{n=1}^{\infty} F_{n}$ generates a dense subalgebra of $A$, so it suffices to verify the Rokhlin property using the sets $F_{n}$ in place of the finite sets of Definition 3.1.

So fix $n$. Let $p \in B$ be the projection

$$
p=1 \otimes 1 \otimes \cdots \otimes 1 \otimes \frac{1}{2}\left(\begin{array}{ll}
1 & 1 \\
1 & 1
\end{array}\right) \otimes 1 \otimes 1 \otimes \cdots,
$$

with the nontrivial entry in the tensor factor in position $n+1$. Then $p$ commutes with everything in $B_{n}$ and $\beta_{0}(p)=1-p$. Now set

$$
q=g-g_{n} \otimes 1+f_{n} \otimes p
$$

Using the relations

$$
u\left(f_{n} \otimes 1\right)=\left(f_{n} \otimes 1\right) u, \quad u\left(g_{n} \otimes 1\right) u^{*}=\left(f_{n}-g_{n}\right) \otimes 1, \quad \text { and } \quad u g u^{*}=1-g,
$$

we get $\alpha(q)=1-q$. Moreover, one easily checks that

$$
g-g_{n} \otimes 1=\sum_{k=n+1}^{\infty} f_{2 k, 2 k} \otimes 1 \quad \text { and } \quad f_{n} \otimes p
$$

commute with every element of $F_{n}$. This shows that $\alpha$ generates an action with the Rokhlin property, proving the claim.

Now we claim that if, in the definition of the Rokhlin property, we take $\varepsilon<1$, then it is not possible to choose $e_{1}$ such that $e_{1} A e_{1}$ is stably isomorphic to a direct summand of $A$. Since the projection $f$ in Theorem 3.2 is just the projection $e_{1}$ in an application of the Rokhlin property, with $\varepsilon>0$ arbitrarily small, this will complete the proof that our example has the properties claimed for it.

Since $A$ can't be written as a direct sum in a nontrivial way, it suffices to rule out stable isomorphism with $A$ and the zero algebra. We can disregard the zero 
algebra, since $\varepsilon<1$ implies $e_{1} \neq 0$. Let $\pi: A \rightarrow \mathbb{C} \oplus \mathbb{C}$ be the quotient map with kernel $K \otimes B$ and which sends $g$ to $(1,0)$. Let $\gamma \in \operatorname{Aut}(\mathbb{C} \oplus \mathbb{C})$ exchange the summands. Then $\gamma \circ \pi=\pi \circ \alpha$. Since $\left\|\alpha\left(e_{1}\right)-\left(1-e_{1}\right)\right\|<1$, we must have $\left\|\gamma\left(\pi\left(e_{1}\right)\right)-\left(1-\pi\left(e_{1}\right)\right)\right\|<1$. This can only happen if $\pi\left(e_{1}\right)$ is either $(1,0)$ or $(0,1)$. In either case, $\pi\left(e_{1} A e_{1}\right) \cong \mathbb{C}$, so $e_{1} A e_{1}$ has at most one nontrivial ideal (namely $\left.e_{1}(K \otimes B) e_{1}\right)$. This is not true of $A$, so $e_{1} A e_{1}$ is not stably isomorphic to $A$. This completes the proof of the claim.

Theorem 3.5. Let $\mathcal{C}$ be any flexible class of separable unital $\mathrm{C}^{*}$-algebras (Definition [1.2). Let $A$ be a unital approximate $\mathcal{C}$-algebra. Let $G$ be a finite group, and let $\alpha: G \rightarrow \operatorname{Aut}(A)$ be an action with the Rokhlin property. Then $C^{*}(G, A, \alpha)$ is again a unital approximate $\mathcal{C}$-algebra.

Proof. Combine Theorem 3.3 and Proposition 1.7

In particular, we have the following result.

Corollary 3.6. Let $A$ be a separable unital $\mathrm{C}^{*}$-algebra, let $G$ be a finite group, and let $\alpha: G \rightarrow \operatorname{Aut}(A)$ have the Rokhlin property.

(1) If $A$ is a unital AI algebra, as defined in Example 2.2 then $C^{*}(G, A, \alpha)$ is a unital AI algebra.

(2) If $A$ is a unital AT algebra, as defined in Example 2.3, then $C^{*}(G, A, \alpha)$ is a unital AT algebra.

(3) If $A$ is a unital AD algebra, as defined in Example 2.4 then $C^{*}(G, A, \alpha)$ is a unital $\mathrm{AD}$ algebra.

(4) If $A$ is a unital countable direct limit of one dimensional noncommutative CW complexes (Definition 2.5), then so is $C^{*}(G, A, \alpha)$.

Proof. The relevant classes are flexible by Examples 2.2, 2.3, 2.4, and 2.8, so we may apply Theorem 3.5

The same conclusion holds for the other flexible classes given in Section 2

We now want to prove similar results for simple unital AH algebras with slow dimension growth and real rank zero, and for Kirchberg algebras satisfying the Universal Coefficient Theorem. These results require preparation.

Proposition 3.7. Let $A$ be a separable nuclear unital $\mathrm{C}^{*}$-algebra, and suppose that $A / I$ satisfies the Universal Coefficient Theorem for every ideal $I \subset A$. Let $G$ be a finite group, and let $\alpha: G \rightarrow \operatorname{Aut}(A)$ have the Rokhlin property. Then $C^{*}(G, A, \alpha) / J$ satisfies the Universal Coefficient Theorem for every ideal $J \subset C^{*}(G, A, \alpha)$.

Proof. Take $\mathcal{C}$ to be the set of all quotients of $A$. The finite saturation of $\mathcal{C}$ consists of finite direct sums of unital $\mathrm{C}^{*}$-algebras which are stably isomorphic to quotients of $A$. All quotients of such algebras are again algebras of the same form. So $C^{*}(G, A, \alpha)$ is a unital local $\mathcal{C}$-algebra by Theorem 3.3. Let $J \subset C^{*}(G, A, \alpha)$ be an ideal. Then $C^{*}(G, A, \alpha) / J$ is also a unital local $\mathcal{C}$-algebra by Lemma 1.6(3). The algebras in the finite saturation of $\mathcal{C}$, and their quotients, are all nuclear and all satisfy the Universal Coefficient Theorem. It now follows from Theorem 1.1 of [10] that $C^{*}(G, A, \alpha) / J$ satisfies the Universal Coefficient Theorem.

Remark 3.8. Similar arguments can be used to show that, for example, if $G$ is finite, $\alpha: G \rightarrow \operatorname{Aut}(A)$ has the Rokhlin property, and $K_{0}(A / I)=0$ for every ideal $I \subset A$, then $K_{0}\left(C^{*}(G, A, \alpha) / J\right)=0$ for every ideal $J \subset C^{*}(G, A, \alpha)$. However, 
at least for simple $A$, much more is known: $K_{*}\left(A^{G}\right) \rightarrow K_{*}(A)$ is injective by Theorem 3.13 of [16], and $K_{*}\left(A^{G}\right) \cong K_{*}\left(C^{*}(G, A, \alpha)\right)$ because $A^{G}$ is isomorphic to a corner in $C^{*}(G, A, \alpha)$ and $C^{*}(G, A, \alpha)$ is simple.

Corollary 3.9. Let $A$ be a simple separable nuclear unital $\mathrm{C}^{*}$-algebra which satisfies the Universal Coefficient Theorem, let $G$ be a finite group, and let $\alpha: G \rightarrow$ $\operatorname{Aut}(A)$ have the Rokhlin property. Then $C^{*}(G, A, \alpha)$ satisfies the Universal Coefficient Theorem.

Theorem 3.10. Let $A$ be a simple unital AH algebra with slow dimension growth and real rank zero, let $G$ be a finite group, and let $\alpha: G \rightarrow \operatorname{Aut}(A)$ have the Rokhlin property. Then $C^{*}(G, A, \alpha)$ is a simple unital AH algebra with slow dimension growth and real rank zero.

Proof. The $\mathrm{C}^{*}$-algebra $A$ has tracial rank zero by Corollary 6.2 .5 of [19]. Therefore $C^{*}(G, A, \alpha)$ has tracial rank zero by Theorem 2.2 of 28 . Also, $C^{*}(G, A, \alpha)$ is simple (by Remark 1.4 and Corollary 1.6 of [28]), separable, and nuclear, and satisfies the Universal Coefficient Theorem (by Corollary 3.9). Therefore the classification theorem of [20, in the form given in Proposition 3.7 of [29], implies that $C^{*}(G, A, \alpha)$ is a simple unital AH algebra with slow dimension growth and real rank zero.

Unlike the results in Corollary 3.6, the proof requires the additional assumptions of simplicity and real rank zero.

A similar result holds for Kirchberg algebras.

Corollary 3.11. Let $A$ be a unital Kirchberg algebra (a simple, separable, nuclear, and purely infinite $\mathrm{C}^{*}$-algebra) satisfying the Universal Coefficient Theorem, let $G$ be a finite group, and let $\alpha: G \rightarrow \operatorname{Aut}(A)$ have the Rokhlin property. Then $C^{*}(G, A, \alpha)$ is a unital Kirchberg algebra satisfying the Universal Coefficient Theorem.

Proof. It is well known that the crossed product is nuclear. The action $\alpha$ is outer, by Lemma 1.5 of [28. Therefore $C^{*}(G, A, \alpha)$ is a unital Kirchberg algebra, by Theorem 3 of [17. It satisfies the Universal Coefficient Theorem by Corollary 3.9.

\section{Further PROPERTIES PRESERVEd By CROSSED PRODUCTS By ACTIONS With THE ROKHLIN PROPERTY}

In this section, we consider preservation of real and stable rank and related properties, such as approximate divisibility, isometric richness, and having the order on projections be determined by traces. We also prove that all tracial states on a crossed product by a finite group action with the Rokhlin property are induced from invariant tracial states on the original algebra.

Proposition 4.1. Let $A$ be a separable unital $\mathrm{C}^{*}$-algebra, let $G$ be a finite group, and let $\alpha: G \rightarrow \operatorname{Aut}(A)$ have the Rokhlin property.

(1) If $A$ has stable rank one, then so does $C^{*}(G, A, \alpha)$.

(2) If $A$ has real rank zero, then so does $C^{*}(G, A, \alpha)$.

Proof. For (11), let $\mathcal{C}$ be the class of all separable unital $\mathrm{C}^{*}$-algebras with stable rank one. We claim that $\mathcal{C}$ is finitely saturated. Conditions (1) and (2) are obvious, and Condition (3) is Theorem 3.3 of [31]. For Condition (4) (corners), we observe that 
$p A p$ is stably isomorphic to the ideal it generates, and use Theorems 3.6 and 4.4 of [31. This proves the claim. It further follows from Theorem 4.3 of 31] that $\mathcal{C}$ is closed under passage to quotients. Theorem 3.3 now implies that for every finite set $F \subset C^{*}(G, A, \alpha)$ and every $\varepsilon>0$, there is $B \in \mathcal{C}$ and a unital homomorphism $\varphi: B \rightarrow C^{*}(G, A, \alpha)$ such that $\operatorname{dist}(a, \varphi(B))<\varepsilon$ for all $a \in F$. This clearly implies that $C^{*}(G, A, \alpha)$ has stable rank one.

For (2), the same argument applies, using the class of separable unital $\mathrm{C}^{*}$ algebras with real rank zero, and using Theorems 2.5, 2.10, and 3.14 of [7].

One expects that a weaker condition on the action than the Rokhlin property should suffice for Proposition 4.1. Compare with the results of 24] for crossed products by $\mathbb{Z}$, and analogous results of [1] for the case of finite groups acting on simple $\mathrm{C}^{*}$-algebras. However, the proposition fails if no condition at all is put on the action. See Example 8.2.1 of [3] for stable rank one, and Example 9 of [13] for real rank zero.

The same proof applies to finite stable rank in place of stable rank one, with appropriate changes in the justification of finite saturation. However, in this case, no condition on $\alpha$ is needed. See Theorem 2.2 and Example 2.1 of [18. For finite real rank, we do not know enough about the real rank of corners and matrix algebras.

Recall (see 4.1 of [9]) that a unital $\mathrm{C}^{*}$-algebra $A$ is said to be isometrically rich if the set of one-sided invertible elements is dense in $A$. This is a weakening of stable rank one which holds for purely infinite simple $\mathrm{C}^{*}$-algebras. It is stronger than extremal richness, as defined at the beginning of Section 3 of $[8]$.

Proposition 4.2. Let $A$ be an isometrically rich unital $\mathrm{C}^{*}$-algebra, let $G$ be a finite group, and let $\alpha: G \rightarrow \operatorname{Aut}(A)$ have the Rokhlin property. Then $C^{*}(G, A, \alpha)$ is isometrically rich.

For the proof, we can't use results about finitely saturated classes. The problem is that the direct sum of two unital isometrically rich $\mathrm{C}^{*}$-algebras is generally not isometrically rich. If $a \in A$ is right invertible but not invertible, and $b \in B$ is left invertible but not invertible, then $(a, b) \in A \oplus B$ can't be approximated by one-sided invertible elements.

Proof of Proposition 4.2. Let $a \in C^{*}(G, A, \alpha)$, and let $\varepsilon>0$. Apply Theorem 3.2 with $\frac{1}{2} \varepsilon$ in place of $\varepsilon$ and with $S=\{a\}$, obtaining $\rho: M_{n} \otimes f A f \rightarrow C^{*}(G, A, \alpha)$ and $b \in M_{n} \otimes f A f$ such that $\|\rho(b)-a\|<\frac{1}{2} \varepsilon$.

We claim that $M_{n} \otimes f A f$ is isometrically rich. Proposition 4.5 of [9] provides an extremally rich primitive $\mathrm{C}^{*}$-algebra $B$ and a surjective homomorphism $\pi: B \rightarrow A$. Choose $b \in B$ with $b \geq 0$ such that $\pi(b)=f$. Then $\overline{b B b}$ is extremally rich by Theorem 3.5 of [8], and is still primitive; moreover, $\pi$ restricts to a surjective homomorphism from $\overline{b B b}$ to $f A f$. Tensor with $\operatorname{id}_{M_{n}}$ to get a surjective homomorphism from $M_{n} \otimes \overline{b B b}$ to $M_{n} \otimes f A f$. The algebra $M_{n} \otimes \overline{b B b}$ is extremally rich by Theorem 4.5 of [8], and is still primitive. So Proposition 4.5 of [9] implies that $M_{n} \otimes f A f$ is isometrically rich, proving the claim.

Accordingly, there is a one-sided invertible element $c \in M_{n} \otimes f A f$ such that $\|c-b\|<\frac{1}{2} \varepsilon$. Then $\rho(c)$ is one-sided invertible in $\rho\left(M_{n} \otimes f A f\right)$, and hence also in $C^{*}(G, A, \alpha)$. Also, $\|\rho(c)-a\|<\varepsilon$. 
Corollary 4.3. Let $A$ be an extremally rich unital prime $\mathrm{C}^{*}$-algebra, let $G$ be a finite group, and let $\alpha: G \rightarrow \operatorname{Aut}(A)$ have the Rokhlin property. Then $C^{*}(G, A, \alpha)$ is isometrically rich.

Proof. Using the condition in Theorem 1.1(ii) of [8], one sees that quasi-invertible elements in $A$ are one-sided invertible. So $A$ is isometrically rich, and Proposition 4.2 applies.

Question 4.4. Let $A$ be an extremally rich unital $\mathrm{C}^{*}$-algebra (not necessarily prime), let $G$ be a finite group, and let $\alpha: G \rightarrow \operatorname{Aut}(A)$ have the Rokhlin property. Does it follow that $C^{*}(G, A, \alpha)$ is extremally rich?

The methods of this paper appear to break down, because local approximation by extremally rich $\mathrm{C}^{*}$-algebras does not necessarily imply extremal richness. In fact, in Example 5.3 of $\left[8\right.$ there is a direct system of extremally rich $\mathrm{C}^{*}$-algebras, with injective unital maps, such that the direct limit is not extremally rich.

The following result was obtained, using different methods (and for compact groups), in Corollary 3.4(2) of [15]. We include the proof here as an example of our methods.

Proposition 4.5. Let $A$ be a separable unital $\mathrm{C}^{*}$-algebra which is approximately divisible in the sense of [6], let $G$ be a finite group, and let $\alpha: G \rightarrow \operatorname{Aut}(A)$ have the Rokhlin property. Then $C^{*}(G, A, \alpha)$ is approximately divisible.

Proof. Take $\mathcal{C}$ to be the collection of all separable unital approximately divisible $\mathrm{C}^{*}$-algebras. This class has approximate quotients (Definition 1.2), because in fact every quotient of an approximately divisible $\mathrm{C}^{*}$-algebra is easily seen to be approximately divisible. So $C^{*}(G, A, \alpha)$ is a unital local $\mathcal{C}$-algebra by Theorem 3.3

It is immediate that $\mathcal{C}$ is closed under isomorphism. It is easy to check that $\mathcal{C}$ is closed under direct sums and tensoring with $M_{n}$, and $\mathcal{C}$ is closed under passing to corners by Corollary 2.9 of $[6$. So $\mathcal{C}$ is finitely saturated.

To show that $C^{*}(G, A, \alpha)$ is approximately divisible, let $F \subset C^{*}(G, A, \alpha)$ be finite and let $\varepsilon>0$. Choose a unital approximately divisible $\mathrm{C}^{*}$-algebra $B$ and a unital homomorphism $\varphi: B \rightarrow A$ such that $\operatorname{dist}(a, \varphi(B))<\frac{1}{3} \varepsilon$ for all $a \in F$. Choose a finite set $S \subset B$ such that $\operatorname{dist}(a, \varphi(S))<\frac{1}{3} \varepsilon$ for all $a \in F$. Passing to $B / \operatorname{ker}(\varphi)$, we may assume $\varphi$ is injective. Choose a unital completely noncommutative finite dimensional $\mathrm{C}^{*}$-algebra $D \subset B$ such that $\|[b, x]\|<\frac{1}{3} \varepsilon$ for all $x \in S$ and all $b \in D$ with $\|b\| \leq 1$. Then $\varphi(D) \subset A$ is a unital completely noncommutative finite dimensional $\mathrm{C}^{*}$-algebra such that $\|[b, x]\|<\varepsilon$ for all $x \in F$ and all $b \in \varphi(D)$ with $\|b\| \leq 1$. Thus $C^{*}(G, A, \alpha)$ is approximately divisible.

We next consider the property that the order on projections over $A$ be determined by traces.

Notation 4.6. Let $A$ be a unital $\mathrm{C}^{*}$-algebra. We denote by $T(A)$ the set of all tracial states on $A$, equipped with the weak* topology. For any element of $T(A)$, we use the same letter for its standard extension to $M_{n}(A)$ for arbitrary $n$, and to $M_{\infty}(A)=\bigcup_{n=1}^{\infty} M_{n}(A)$ (no closure).

Definition 4.7. Let $A$ be a simple unital $\mathrm{C}^{*}$-algebra. We say that the order on projections over $A$ is determined by traces if whenever $p, q \in M_{\infty}(A)$ are projections such that $\tau(p)<\tau(q)$ for all $\tau \in T(A)$, then $p \precsim q$. 
This is Blackadar's Second Fundamental Comparability Question for $M_{\infty}(A)$. See 1.3.1 in [4].

Proposition 4.8. Let $A$ be a simple separable unital $\mathrm{C}^{*}$-algebra such that the order on projections over $A$ is determined by traces in the sense of Definition 4.7 Let $G$ be a finite group, and let $\alpha: G \rightarrow \operatorname{Aut}(A)$ have the Rokhlin property. Then the order on projections over $C^{*}(G, A, \alpha)$ is determined by traces.

Proof. The crossed product is simple, by Remark 1.4 and Corollary 1.6 of [28]. So Definition 4.7 applies.

Note that $M_{n}\left(C^{*}(G, A, \alpha)\right) \cong C^{*}\left(G, M_{n} \otimes A, \operatorname{id}_{M_{n}} \otimes \alpha\right)$, and $\operatorname{id}_{M_{n}} \otimes \alpha$ also has the Rokhlin property. It therefore suffices to verify the condition of Definition 4.7 for projections in $C^{*}(G, A, \alpha)$.

Take $\mathcal{C}=\{A\}$. The finite saturation of $\mathcal{C}$ consists of finite direct sums of unital $\mathrm{C}^{*}$-algebras which are stably isomorphic to $A$. All quotients of such algebras are again algebras of the same form. So $C^{*}(G, A, \alpha)$ is a unital local $\mathcal{C}$-algebra by Theorem 3.3 .

We finish the proof via an adaptation of a standard argument. Let $p, q \in$ $C^{*}(G, A, \alpha)$, and suppose it is not the case that $p \precsim q$. We need to find a tracial state on $C^{*}(G, A, \alpha)$ such that $\tau(p) \geq \tau(q)$. Let $\left\{x_{1}, x_{2}, \ldots\right\}$ be a countable dense subset of $C^{*}(G, A, \alpha)$. For each $n \geq 1$, choose a $\mathrm{C}^{*}$-algebra $B_{n}$ in the finite saturation of $\mathcal{C}$ and a unital homomorphism $\varphi_{n}: B_{n} \rightarrow A$ such that $\operatorname{dist}\left(x, \varphi_{n}\left(B_{n}\right)\right)<2^{-n}$ for all $x \in\left\{p, q, x_{1}, x_{2}, \ldots, x_{n}\right\}$. From the description of the finite saturation of $\mathcal{C}$ given above, we may assume that each $\varphi_{n}$ is injective. We therefore treat $B_{n}$ as a unital subalgebra of $C^{*}(G, A, \alpha)$, and delete $\varphi_{n}$ from the notation. We further write

$$
B_{n}=B_{1, n} \oplus B_{2, n} \oplus \cdots \oplus B_{k(n), n}
$$

for unital $\mathrm{C}^{*}$-algebras $B_{j, n}$ which are stably isomorphic to $A$. Each $B_{j, n}$ is a corner of a matrix algebra over $A$, so the order on projections over $B_{j, n}$ is determined by traces.

Since $\operatorname{dist}\left(p, B_{n}\right), \operatorname{dist}\left(q, B_{n}\right)<2^{-n} \leq \frac{1}{2}$, there exist projections $e_{n}, f_{n} \in B_{n}$ such that $e_{n} \sim p$ and $f_{n} \sim q$. Moreover, we may arrange that $\lim _{n \rightarrow \infty} e_{n}=p$ and $\lim _{n \rightarrow \infty} f_{n}=q$. Write

$$
e_{n}=\left(e_{1, n}, e_{2, n}, \ldots, e_{k(n), n}\right) \quad \text { and } \quad f_{n}=\left(f_{1, n}, f_{2, n}, \ldots, f_{k(n), n}\right)
$$

with $e_{j, n}, f_{j, n} \in B_{j, n}$. It is not the case that $e_{n} \precsim f_{n}$, so there is $j(n)$ such that it is not the case that $e_{j(n), n} \precsim f_{j(n), n}$. Therefore there is a tracial state $\tau_{n}$ on $B_{j(n), n}$ such that $\tau_{n}\left(e_{j(n), n}\right) \geq \tau_{n}\left(f_{j(n), n}\right)$. Regard $\tau_{n}$ as a tracial state on $B_{n}$ by taking $\tau_{n}=0$ on the other summands of $B_{n}$. By the Hahn-Banach Theorem, there is a state $\omega_{n}$ on $C^{*}(G, A, \alpha)$ whose restriction to $B_{n}$ is $\tau_{n}$.

Let $\tau$ be a state which is a weak* limit point of the states $\omega_{n}$.

We claim that $\tau$ is tracial. Let $a, b \in C^{*}(G, A, \alpha)$, and let $\varepsilon>0$. We may assume $\varepsilon<1$. Set $M=1+\max (\|a\|,\|b\|)$. Choose $n_{0}$ such that $2^{-n_{0}}<\frac{1}{6} M^{-1} \varepsilon$ and so large that there are $k, l \in\left\{1,2, \ldots, n_{0}\right\}$ such that

$$
\left\|x_{k}-a\right\|<\frac{1}{6} M^{-1} \varepsilon \quad \text { and } \quad\left\|x_{l}-b\right\|<\frac{1}{6} M^{-1} \varepsilon .
$$

Note that $\left\|x_{k}\right\|,\left\|x_{l}\right\|<M$. Therefore

$$
\left\|a b-x_{k} x_{l}\right\| \leq\left\|a-x_{k}\right\| \cdot\|b\|+\left\|x_{k}\right\| \cdot\left\|b-x_{l}\right\|<\left(\frac{1}{6} M^{-1} \varepsilon\right) M+M\left(\frac{1}{6} M^{-1} \varepsilon\right)=\frac{1}{3} \varepsilon .
$$


For all $n \geq n_{0}$, we have $\omega_{n}\left(x_{k} x_{l}\right)=\omega_{n}\left(x_{l} x_{k}\right)$, so

$$
\left|\omega_{n}(a b)-\omega_{n}(b a)\right| \leq 2\left\|a b-x_{k} x_{l}\right\|<\frac{2}{3} \varepsilon .
$$

Choose $n \geq n_{0}$ such that

$$
\left|\tau(a b)-\omega_{n}(a b)\right|<\frac{1}{6} \varepsilon \quad \text { and } \quad\left|\tau(b a)-\omega_{n}(b a)\right|<\frac{1}{6} \varepsilon .
$$

Then

$$
|\tau(a b)-\tau(b a)|<\frac{1}{3} \varepsilon+\left|\omega_{n}(a b)-\omega_{n}(b a)\right|<\frac{1}{3} \varepsilon+\frac{2}{3} \varepsilon=\varepsilon .
$$

Since $\varepsilon>0$ is arbitrary, the claim follows.

We complete the proof by showing that $\tau(p) \geq \tau(q)$. Let $\varepsilon>0$. Choose $n_{0}$ such that $n \geq n_{0}$ implies

$$
\left\|e_{n}-p\right\|<\frac{1}{4} \varepsilon \quad \text { and } \quad\left\|f_{n}-q\right\|<\frac{1}{4} \varepsilon .
$$

Choose $n \geq n_{0}$ such that

$$
\left|\tau(p)-\omega_{n}(p)\right|<\frac{1}{4} \varepsilon \quad \text { and } \quad\left|\tau(q)-\omega_{n}(q)\right|<\frac{1}{4} \varepsilon .
$$

Then

$$
\left|\tau(p)-\omega_{n}\left(e_{n}\right)\right| \leq\left|\tau(p)-\omega_{n}(p)\right|+\left\|p-e_{n}\right\|<\frac{1}{4} \varepsilon+\frac{1}{4} \varepsilon=\frac{1}{2} \varepsilon,
$$

and similarly $\left|\tau(q)-\omega_{n}\left(f_{n}\right)\right|<\frac{1}{2} \varepsilon$. Since $\omega_{n}\left(e_{n}\right) \geq \omega_{n}\left(f_{n}\right)$, we conclude that $\tau(p)>\tau(q)-\varepsilon$. Since $\varepsilon>0$ is arbitrary, the proof is complete.

The following result does not use the local approximation result of Theorem 3.3 , However, we have not found it in the literature, and it is included here because the statement seems related to other results here. The assumption that the action have the Rokhlin property is stronger than necessary, at least when the $\mathrm{C}^{*}$-algebra is simple: the tracial Rokhlin property suffices. See Proposition 5.7 of [1].

Proposition 4.9. Let $A$ be a separable unital $C^{*}$-algebra. Let $G$ be a finite group, and let $\alpha: G \rightarrow \operatorname{Aut}(A)$ have the Rokhlin property. Then the restriction map defines a bijection from $T\left(C^{*}(G, A, \alpha)\right)$ (see Notation 4.6) to the set $T(A)^{G}$ of $G$-invariant tracial states on $A$.

Proof. For $g \in G$, let $u_{g} \in C^{*}(G, A, \alpha)$ be the standard unitary in the crossed product. Let $E: C^{*}(G, A, \alpha) \rightarrow A$ be the standard conditional expectation, given by $E\left(\sum_{g \in G} a_{g} u_{g}\right)=a_{1}$ when $a_{g} \in A$ for $g \in G$.

We claim that the map $\tau \mapsto \tau \circ E$ is an inverse of the restriction map. First, if $\tau \in T(A)^{G}$, one easily checks that $\tau \circ E$ is a tracial state on $C^{*}(G, A, \alpha)$, and it is immediate that its restriction to $A$ is $\tau$.

So suppose $\tau \in T\left(C^{*}(G, A, \alpha)\right)$. Proving that $\tau=\left(\left.\tau\right|_{A}\right) \circ E$ is equivalent to proving that $\tau\left(a u_{g}\right)=0$ for $g \in G \backslash\{1\}$ and $a \in A$. Let $\varepsilon>0$. Choose projections $e_{h} \in A$ for $h \in G$ according to the Rokhlin property (Definition 3.1), with

$$
\delta=\frac{\varepsilon}{(1+\|a\|) \operatorname{card}(G)}
$$

in place of $\varepsilon$ and with $\{a\}$ in place of $F$. For any $h \in G$, we then have $e_{h} u_{g} e_{h}=$ $e_{h}\left(u_{g} e_{h} u_{g}^{*}-e_{g h}\right) u_{g}+e_{h} e_{g h} u_{g}$. Since $g \neq 1$, we have $e_{h} e_{g h}=0$, whence $\left\|e_{h} u_{g} e_{h}\right\|<\delta$. 
Now

$$
\begin{aligned}
\left|\tau\left(a u_{g}\right)\right| & \leq \sum_{h \in G}\left|\tau\left(a u_{g} e_{h}^{2}\right)\right|=\sum_{h \in G}\left|\tau\left(e_{h} a u_{g} e_{h}\right)\right| \leq \sum_{h \in G}\left(\left\|e_{h} a-a e_{h}\right\|+\left|\tau\left(a e_{h} u_{g} e_{h}\right)\right|\right) \\
& \leq \sum_{h \in G}\left(\left\|e_{h} a-a e_{h}\right\|+\|a\| \cdot\left\|e_{h} u_{g} e_{h}\right\|\right)<\operatorname{card}(G)(1+\|a\|) \delta=\varepsilon .
\end{aligned}
$$

Since $\varepsilon>0$ is arbitrary, we conclude that $\tau\left(a u_{g}\right)=0$.

Corollary 4.10. Let $A$ be a separable unital $\mathrm{C}^{*}$-algebra with a unique tracial state. Let $G$ be a finite group, and let $\alpha: G \rightarrow \operatorname{Aut}(A)$ have the Rokhlin property. Then $C^{*}(G, A, \alpha)$ has a unique tracial state.

Proof. Apply Proposition 4.9, noting that the tracial state on $A$ is necessarily $G$ invariant.

\section{REFERENCES}

[1] D. Archey, Crossed product $C^{*}$-algebras by finite group actions with a generalized tracial Rokhlin property, Ph.D. Thesis, University of Oregon, Eugene, 2008.

[2] B. Blackadar, Shape theory for $C^{*}$-algebras, Math. Scand. 56(1985), 249-275.

[3] B. Blackadar, Symmetries of the CAR algebra, Ann. Math. (2) 131(1990), 589-623.

[4] B. Blackadar, Comparison theory for simple $C^{*}$-algebras, pages 21-54 in: Operator Algebras and Applications, D. E. Evans and M. Takesaki (eds.) (London Math. Soc. Lecture Notes Series no. 135), Cambridge University Press, Cambridge, New York, 1988.

[5] B. Blackadar, Semiprojectivity in simple $C^{*}$-algebras, pages 1-17 in: Operator Algebras and Applications (Adv. Stud. Pure Math. vol. 38), Math. Soc. Japan, Tokyo, 2004.

[6] B. Blackadar, A. Kumjian, and M. Rørdam, Approximately central matrix units and the structure of non-commutative tori, K-Theory 6(1992), 267-284.

[7] L. G. Brown and G. K. Pedersen, $C^{*}$-algebras of real rank zero, J. Funct. Anal. 99(1991), 131-149.

[8] L. G. Brown and G. K. Pedersen, On the geometry of the unit ball of a $C^{*}$-algebra, J. reine angew. Math. 469(1995), 113-147.

[9] L. G. Brown and G. K. Pedersen, Ideal structure and $C^{*}$-algebras of low rank, Math. Scand. 100(2007), 5-33.

[10] M. Dadarlat, Some remarks on the universal coefficient theorem in KK-theory, pages 65-74 in: Operator algebras and mathematical physics (Constanţa, 2001), Theta, Bucharest, 2003.

[11] S. Echterhoff, W. Lück, N. C. Phillips, and S. Walters, The structure of crossed products of irrational rotation algebras by finite subgroups of $\mathrm{SL}_{2}(\mathbb{Z})$, preprint (arXiv: math.OA/0609784).

[12] S. Eilers, T. A. Loring, and G. K. Pedersen, Stability of anticommutation relations. An application of noncommutative $C W$ complexes, J. reine angew. Math. 499(1998), 101-143.

[13] G. A. Elliott, A classification of certain simple $C^{*}$-algebras, pages 373-385 in: Quantum and Non-Commutative Analysis, H. Araki etc. (eds.), Kluwer, Dordrecht, 1993.

[14] E. C. Gootman and A. J. Lazar, Applications of noncommutative duality to crossed product $C^{*}$-algebras determined by an action or coaction, Proc. London Math. Soc. (3) 59(1989), 593-624.

[15] I. Hirshberg and W. Winter, Rokhlin actions and self-absorbing $C^{*}$-algebras, Pacific J. Math. 233(2007), 125-143.

[16] M. Izumi, Finite group actions on $C^{*}$-algebras with the Rohlin property. I, Duke Math. J. 122(2004), 233-280.

[17] J. A. Jeong, Purely infinite simple $C^{*}$-crossed products, Proc. Amer. Math. Soc. 123(1995), 3075-3078.

[18] J. A. Jeong, H. Osaka, N. C. Phillips, and T. Teruya, Cancellation for inclusions of $C^{*}$ algebras of finite depth, Indiana Univ. Math. J., to appear.

[19] H. Lin, An Introduction to the Classification of Amenable $C^{*}$-algebras, World Scientific, River Edge NJ, 2001.

[20] H. Lin, Classification of simple $C^{*}$-algebras with tracial topological rank zero, Duke Math. J. 125(2005), 91-119. 
[21] T. A. Loring, Lifting Solutions to Perturbing Problems in $C^{*}$-Algebras, Fields Institute Monographs no. 8, American Mathematical Society, Providence RI, 1997.

[22] M. Nagisa, Single generation and rank of $C^{*}$-algebras, pages 135-143 in: Operator Algebras and Applications, Adv. Stud. Pure Math. vol. 38, Math. Soc. Japan, Tokyo, 2004.

[23] C. L. Olsen and W. R. Zame, Some $C^{*}$-algebras with a single generator, Trans. Amer. Math. Soc. 215(1976), 205-217.

[24] H. Osaka and N. C. Phillips, Stable and real rank for crossed products by automorphisms with the tracial Rokhlin property, Ergod. Th. Dynam. Sys. 26(2006), 1579-1621.

[25] H. Osaka and N. C. Phillips, Crossed products of simple $C^{*}$-algebras with tracial rank one by actions with the tracial Rokhlin property, in preparation.

[26] C. Pasnicu and N. C. Phillips, in preparation.

[27] G. K. Pedersen, Pullback and pushout constructions in $C^{*}$-algebra theory, J. Funct. Anal. 167(1999), 243-344.

[28] N. C. Phillips, The tracial Rokhlin property for actions of finite groups on $C^{*}$-algebras, preprint (arXiv: math.OA/0609782).

[29] N. C. Phillips, Every simple higher dimensional noncommutative torus is an AT algebra, preprint (arXiv: math.OA/0609783).

[30] N. C. Phillips and M. G. Viola, A simple separable exact $C^{*}$-algebra not anti-isomorphic to itself, in preparation.

[31] M. A. Rieffel, Dimension and stable rank in the K-theory of $C^{*}$-algebras, Proc. London Math. Soc. (3) 46(1983), 301-333.

Department of Mathematical Sciences, Ritsumeikan University, Kusatsu, Shiga, 5258577 JAPAN

E-mail address: osaka@se.ritsumei.ac.jp

Department of Mathematics, University of Oregon, Eugene OR 97403-1222, USA.

E-mail address: ncp@darkwing.uoregon.edu 